\title{
Erhöhung der Oberflächenkompatibilität von PEEK als Matrix- werkstoff für kohlenstoffaserverstärkte Verbundwerkstoffe: Calciumphosphatbeschichtung aus übersättigter Lösung
}

\author{
S.-W. Ha', M. Kirch'1, F. Birchler', R. Reber', E. Wintermantel', \\ M. Guecheva ${ }^{2}$ und $H$. Vonmont ${ }^{2}$ \\ ${ }^{1}$ Professur für Biokompatible Werkstoffe und Bauweisen, ETH Zürich, Schweiz \\ 2 Eidgenössische Materialprüfungs- und Forschungsanstalt (EMPA), Dübendorf, Schweiz
}

\section{Einleitung}

Die Beschichtung von lasttragenden, metallischen Hüftgelenksendoprothesen mit Hydroxylapatit zur Erhöhung des primären Anwachsverhaltens vor allem von pathologischem Knochengewebe ist heute Stand der Technik $(1,2)$. Eines der kommerziell eingesetzten Beschichtungsverfahren stellt das Plasmaspritzverfahren dar. Aufgrund der auftretenden hohen Temperaturen und der hohen mechanischen Substratbelastung muss der Plasmaspritzprozess für die Anwendung auf thermoplastischen Faserverbundwerkstoffen optimiert werden (3). Die Beschíchtung aus übersättigter Calciumphosphatlösung stellt eine schonende Möglichkeit dar, um eine erhöhte Biokompatibilität von thermoplastischen Polymeren und Faserverbundwerkstoffen zu erreichen.

\section{Material und Methoden}

Aus spritzgegossenem Polyetheretherketon (PEEK) wurden Proben $(20 \mathrm{~mm} * 20 \mathrm{~mm} * 4 \mathrm{~mm})$ zugeschnitten und anschliessend poliert. Die polierten PEEK-Oberflächen wurden in einer $\mathrm{N}_{2} / \mathrm{O}_{2}$-Niederdruck-Plasmakammer (Harrick Plasma Cleaner/Sterilizer PDC 32G) aktiviert. Die Plasmaerzeugung erfolgte mit einem Hochfrequenzgenerator $(10 \mathrm{MHz})$ bei einer Leistung von $100 \mathrm{~W}$. Der Effekt der Oberflächenaktivierung wurde mittels statischer Kontaktwinkelmessung ermittelt. Mittels Neutral-Red Assay (NR) und MTT-Dehydrogenase Aktivität (MTT) wurde der Einfluss der Oberflächenaktivierung nach 10 min Plasmabehandlung - auf die Zellvitalität untersucht. Die Proben wurden hierfür während $3 \mathrm{~h}$ bei $180^{\circ} \mathrm{C} \mathrm{m}$ Heissluftsterilisator sterilisiert und anschliessend während 48h mit MC3T3E1-Zellen (Osteoblasten-Zellinien) inkubiert $\left(37^{\circ} \mathrm{C}, 5 \% \mathrm{CO}_{2}, 95 \%\right.$ relative Lufffeuchtigkeit).

Zur Herstellung der Calciumphosphatlösung wurden zunächst $100 \mathrm{mM}$ Tris und $35 \mathrm{mM} \mathrm{HCl}$ in bidestilliertem Wasser gelöst und anschliessend $0.62 \mathrm{mM} \mathrm{H}_{3} \mathrm{PO}_{4}$ und $1 \mathrm{mM} \mathrm{Ca}(\mathrm{OH})_{2}$ in den hergestellten Puffer dazugegeben. Der Beschichtungsprozess wurde in dieser Lösung, bei $\mathrm{pH} 8$ und bei $\mathrm{T}=37^{\circ} \mathrm{C}$, während 24 Tagen unter angenäherten "constant composition" Bedingungen durchgeführt. Hierfür wurden die Proben täglich einem Lösungswechsel unterzogen. Der Nukleationsprozess erfolgte in einer stark mit Hydroxylapatit (HA) übersättigten Lösung, wozu HA-Pulver (Heyl, Berlin) in bidestilliertem Wasser suspendiert wurde. Auf dem entstehenden HA-Bodensatz wurden die Probe mit der zu beschichtenden Oberfläche nach unten während 7 Tagen dem Nukleationsprozess unterzogen.

Mittels ICP-Alomemissionsspektroskopie (ICP-AES) wurden die Calcium- und Phosphorkonzentrationen der Lösungen in Funktion der Beschichtungszeit ermittelt. Die erzeugten Beschichtungen wurden mittels lichtmikroskopischen (LM) und rasterelektronischen (REM) Untersuchungen sowie mittels Infrarotspektroskopie (FTIR) charakterisiert. Die Zellvitalität auf unbeschichteten und beschichteten PEEKSubstraten wurde mittels MTT-, NR- und BrdU ELISA-Testung untersucht. Die Proben wurden während $3 \mathrm{~h}$ bei $180^{\circ} \mathrm{C}$ im Heissluftsterilisator sterilisiert und anschliessend während $48 \mathrm{~h}$ mit MC3T3E1-Zellen inkubiert $\left(37^{\circ} \mathrm{C}, 5 \% \mathrm{CO}_{2}, 95 \%\right.$ relative Lufffeuchtigkeit).

\section{Resultate}

Oberflächenaktivierung

Die Oberflächenaktivierung im $\mathrm{N}_{2} / \mathrm{O}_{2}$-Niederdruck-Plasma hatte eine signifikante Erniedrigung des Kontaktwinkels zur Folge. Bereits nach einer Plasmaeinwirkung von 5 Sekunden wurde eine Senkung des Kontaktwinkels um $50^{\circ}$ beobachtet (vgl. Abb. 1). Auf die Zellvitalität von Osteoblasten wurde ein tendenziell positiver Effekt der Plasmaaktivierung festgestellt, wie die MTT- und NR-Untersuchungen gezeigt haben ( $\mathrm{vgl}$. Abb. 2).

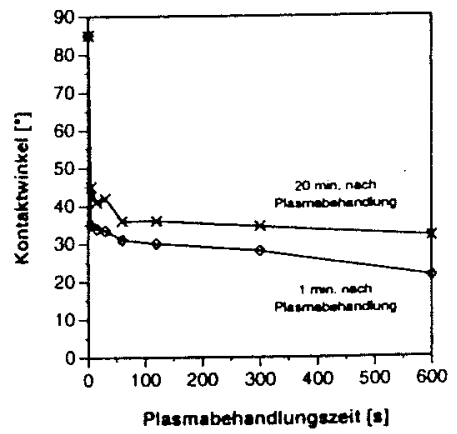

Abb. 1: Bestimmung der Kontaktwinkel nach verschiedenen Plasmabehandlungszeiten. Die beiden Messkurven entsprechen Messungen, die 1 min, bzw. 20 min nach der Plasmaaktivierung durchgeführt wurden.

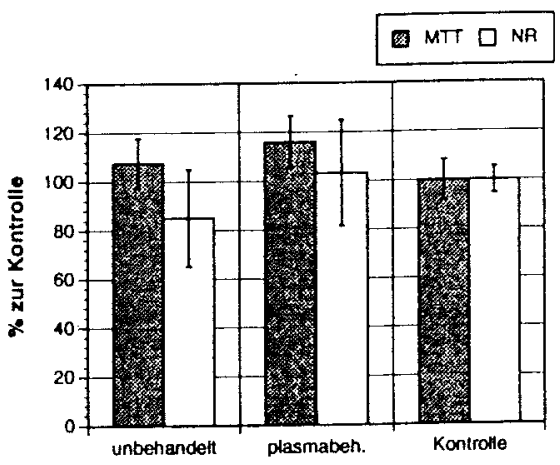

Abb. 2: MTT- und NR-Zellvitalitätstest auf polierten PEEK-Proben nach 48h Besiedlung mit MC3T3E1-Zellen ( $n=3)$. 


\section{- Beschichtung}

LM- und REM-Untersuchungen haben gezeigt, dass nach 24-tägiger Auslagerung in übersättigter Lösung Schichten mit

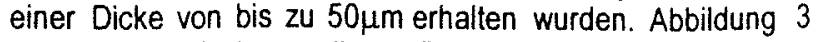
zeigt REM-Aufnahmen dieser Beschichtung, die aus einem Nukleationsrasen und einer Schicht aus plättchenförmigen Kristalliten besteht. Mittels FTIR konnten die charakteristischen $v_{3}-P_{4}$-Schwingungsbanden bei Wellenzahl 1036 und $1089 \mathrm{~cm}^{-1}$ ermittelt werden. Zudem traten bei $873 \mathrm{~cm}^{-1}$, bzw. 1400-1460 $\mathrm{cm}^{-1}$ Banden auf, die $\mathrm{v}_{2}-\mathrm{CO}_{3-}$, bzw. $\mathrm{v}_{3}-\mathrm{CO}_{3-}$ Schwingungsmodi zuzuordnen sind.

ICP-AES Untersuchungen haben gezeigt, dass sowohl eine 7-tägige Nukleation wie auch eine Vorbehandlung der Substratoberfläche in einem $\mathrm{N}_{2} / \mathrm{O}_{2}$-Niederdruck-Plasma die Wachstumsrate der Beschichtung positiv beeinflusst, wie nachfolgende Abbildung 4 zeigt. Bei den Zellkulturversuchen mit MC3T3E1-Zellen wurde auf den aus übersättigter Lösung beschichteten PEEK-Substraten im Vergleich zu den unbeschichteten Oberflächen bei allen drei Testmethoden eine höhere Zellvitalität festgestellt (vgl. Abb. 5).

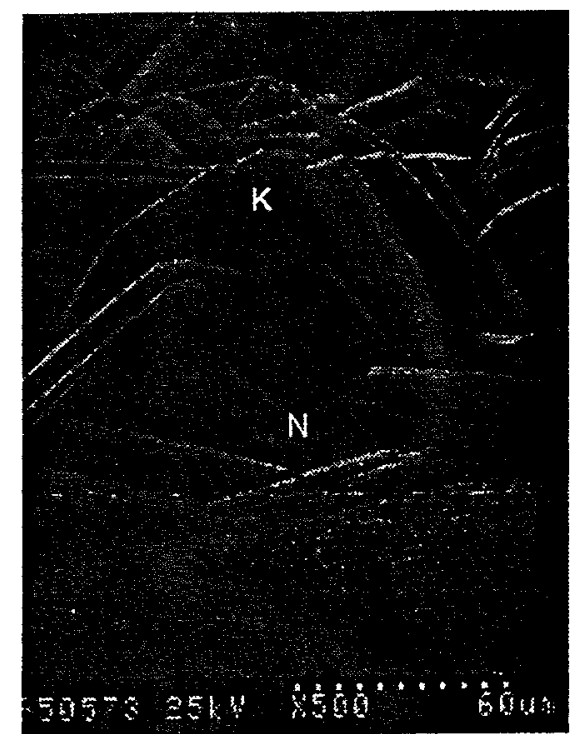

Abb. 3: REM-Aufnahme der aus übersättigter Calciumphosphatlösung hergestellten Beschichtung auf einer unbehandelten, polierten PEEK-Oberfläche nach 7 tägiger Nukleation ( $\mathrm{N}$ : Nukleationsrasen; K: Kristallite)

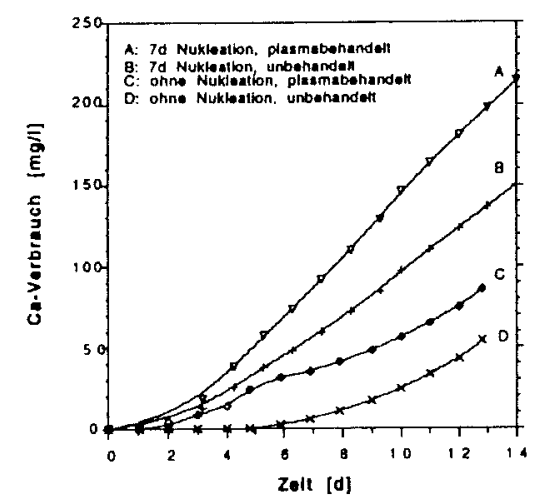

Abb. 4: CaVerbrauch bei der Beschichtung von polierten PEEKSubstraten aus übersättigter Lösung. Die Graphik zeigt den positiven Einfluss der vorgängigen Nukleation und der Plasmaaktivierung auf die Abscheidungsrate.

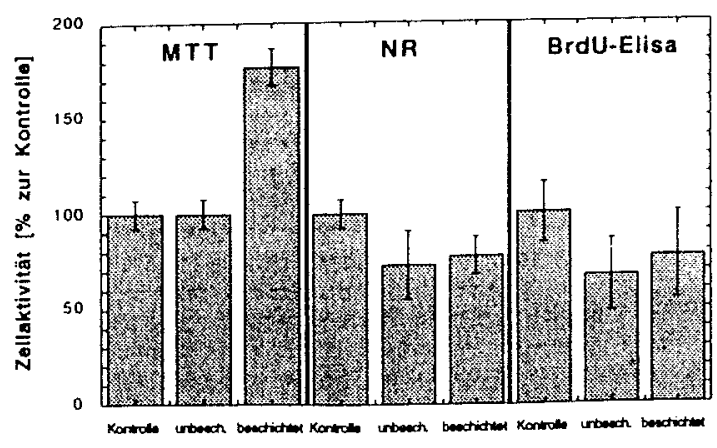

Abb. 5: Einfluss der Beschichtung auf die Zellaktivität von MC3T3E1-Zellen. Im Vergleich zur unbeschichteten Probe wurde auf den mittels Abscheidung aus übersättigter Lösung beschichteten PEEK-Substraten in allen drei Testmethoden eine höhere Vitalität festgestellt $(n=9)$.

\section{Diskussion}

Die Beschichtung von PEEK-Oberflächen in einer an Calciumphosphat übersättigten Lösung führte zur Bildung einer carbonathaltigen Calciumphosphatschicht. Die Kinetik der Schichtbildung konnte dabei durch Oberflächenaktivierung in einem $\mathrm{N}_{2} / \mathrm{O}_{2}$-Niederdruck-Plasma und durch eine vorgängige Nukleation während 7 Tagen gesteigert werden. In $(4,5)$ wird beschrieben, dass das Knochenanwachsverhalten von bioaktiven Werkstoffen mit der Bildung und dem Wachstum einer carbonathaltigen Apatitschicht auf der Werkstoffoberfläche korreliert. Die Bildung dieser Apatitschicht wurde dabei von unterschiedlichen Autoren auf Bioglas- (6) und Silika-Gel-Oberflächen (7) beobachtet und darin die hohe Bioaktivität dieser Werkstoffe begründet. Die Ergebnisse der in der vorliegenden Arbeit durchgeführten Untersuchungen haben gezeigt, dass sich auf der PEEK-Oberfläche eine carbonathaltige Calciumphosphatschicht gebildet hat. Weiterführende Untersuchungen sollten Aufschluss über die Bioaktivität von aus übersättigter Lösung hergestellten Calciumphosphatschichten geben.

\section{Literatur}

(1) de Groot K., Klein C.P.A.T., Wolke J.G.C., de Blieck-Hogervorst J.M.A., Plasma-sprayed coatings of calcium phosphate, 2, CRC Press, Boca Raton, Ann Arbor, Boston, 133-142 (1990)

(2) Gross K.A., Berndt C.C., In vitro testing of plasma-sprayed hydroxyapatite coating, Journal of materials science: Materials in medicine, 5, 219-224 (1994)

(3) Ha S.-W., Mayer J., Koch B., Wintermantel E., Plasma-sprayed hydroxylapatite coating on carbon fiber reinforced thermoplastic composite materials, Joumal of materials science: Materials in medicine, 5, 481-484 (1994)

(4) Daculsi, G., LeGeros, R.Z., Heugehbaert, M., Barbieux, L., Formation of carbonate apatite crystals after implantation of calcium phosphate ceramics, Calcif. Tiss. Int., 46, 20-27 (1990)

(5) Ducheyne, P., Radin, S., King, K., Ishikawa, K., Kim, C.S., In vivo dissolution and precipitation of calcium phosphate phases on biomaterials correlates with in vivo bioactivity, in: Bonfield, $W$., Ha stings, G.W., Tanner, K.E. (eds.), Bioceramics 4, Butterworth Heinemann, London, 135-144 (1991)

(6) Hench, L.L., Andersson, Ö., Bioactive glasses, in: Hench L.L., Wilson, J., An introduction to bioceramics, World Scientific Publishing Co., Singapore, 41-73 (1993)

(7) Li, P., Ohtsuki, C., Kokubo. T., Nakanishi, K., Soga, N., Nakamura, T., Yamamuro, T., Process of formation of bone-like apatite layer on silica gel, Journal of Materials Science: Material in Medicine, 4, 127-131 (1993) 